\title{
The Use of Combined Oral Contraceptives in Women with Multiple Sclerosis
}

\author{
Tikhonovskaya $\mathbf{O}$ and MD Kochetkova $\mathrm{A}^{*}$ \\ Siberian State Medical University, Russia
}

Received: May 7, 2018; Published: May 17, 2018

*Corresponding author: Kochetkova Anastasia, Full Time Postgraduate at the Department of Obstetrics and Gynecology, Siberian State Medical University, Russia

\section{Opinion}

Multiple sclerosis (MS) is an autoimmune disease of the central nervous system, which is characterized by a progressive increase in neurological symptoms. The prevalence of MS in the world has recently increased. This is due both to a real increase in the incidence rate and to the use of modern neuroimaging methods of research. It can be assumed that sex steroid hormones affect the pathogenesis and course of MS because the prevalence of MS among women is 2 times higher than that of men, and in addition, the disease has gender differences. The predominance of women is particularly high among young patients, as well as in the family group.

The course of MS is correlated to a certain extent with the concentrations of sex steroid hormones, so it seems logical to designate combined oral contraceptives (COCs) for the purpose of contraception for women with this disease. We conducted a study aimed at studying the effect of COCs on the course of MS. The study included 55 women with a remitting course of the disease, the mean age of patients was $27.9 \pm 7.3$ (19 to 45 years), $35(63.6 \%)$ women of early reproductive age (19-35 years) and 20 (36.4\%) women of late reproductive age (36-45 years). The average age of debut of the disease was $26.4 \pm 7.9$ (11 to 44 years), the average duration of the disease at the time of the examination was $6.4 \pm 4.8$ (from 1 to 18 years).

Depending On The Administration of Cocs, 3 Groups of Patients Were Allocated: I - never taking COC, II - taking COC prior to onset of MS, III - taking COC after the diagnosis of MS. Patients of Groups II and III received monophasic COCs for contraception. Patients underwent a comprehensive examination, including a standard gynecological examination, neurologic examination and sonography of pelvic organs. Quantitative assessment of functional impairment in MS was carried out according to the extended scale of disability Kurtzke (EDSS - Expanded Disability Status Scale), proposed by John Kurtzke in 1983. Statistical analysis of data was performed using the SPSS Statistics 17.0 software package.
Based on the results of the study, the average duration of using COCs in groups II and III was $3.5 \pm 2.0$ and $3.0 \pm 2.4$ years, respectively. The average EDSS score in the groups of patients taking COCs before the beginning of the MS (II group) and taking COC after diagnosing MS (group III) was $2.4 \pm 1.68$. In the group of patients who never took COCs (group I), the average EDSS score was $3.5 \pm 1.4$ points. Compared to patients who never used COCs, patients who received COCs before and after diagnosis of MS had lower scores on the EDSS scale. Thus, patients with MS showed an adverse side effect of neuroprotective effect of COCs.

It should be noted that before the appointment of COCs, it is necessary to assess the risk of reducing the steroid function of the ovaries in this group of patients. A case-control study of women of early and late reproductive age $(n=75)$ was conducted, with a reliable diagnosis of MS. Quantitative assessment of functional disorders with MS was performed according to the extended scale of disability according to Kurtzke. All patients underwent an ultrasound study of small-box organisms (APLIO XG, Toshiba Medical Systems Corporation, Japan). To assess the hormonal status, serum concentrations of FSH, free testosterone, dihydroepiandrosterone sulfate (DHEA-S), AMG were determined by the enzyme immunoassay. To construct a mathematical model, the method of discriminant analysis was used. Statistical processing of the results was performed using the "SPSS 22.0" package (IBM Inc, USA).

Taking into Account the Obtained data, the Final Mathematical Model for Calculating the Risk of Reducing the Steroid Function of the Ovaries in Patients with ms Assumes the form:

$\mathrm{F}=1,031 * \mathrm{EDSS}+0,149 *$ rate of progression of MS +0.234 *total FAS (follicle antral score) - $(0.020 *$ free testosterone + $0.018 *$ DHEA-S), where $\mathrm{F}$ is the predictive model (the value of the discriminant function). If $\mathrm{F}>0$, this indicates the earliest formation of lower ovarian reserve in women with MS; If $\mathrm{F}<0$, then this pathology is absent. 
I. Example: Patient T., 36 years old. EDSS $=4.0$, the rate of disease progression is 1.2 , the total FAS is 11 , free testosterone is $0.8 \mathrm{pg} / \mathrm{ml}$, DHEA-S is $115 \mu \mathrm{g} / \mathrm{dL}$.With the help of the developed mathematical model, an analysis of the risk of early formation of the lower ovaian reserve in this patient was conducted:

$\mathrm{F}=1,031 * 4,0+0,149 * 1,2+0,234 * 11-(0,020 * 0,8+0,018$

$* 115)=4,79$, which indicates a risk of early reduction of steroid function of the ovaries. When manufacturing the mathematical model, the decrease in the reduction of the ovarian reserve was taken into account, as FSH $>14 \mathrm{IU} / \mathrm{ml}, \mathrm{B}<8 \mathrm{~cm} 3$ [Nazarenko TA,
Krasnopolskaya KV, 2013]. The sensitivity of the developed model is $76 \%$, specificity is $94 \%$. The use prognostic model for assessing the steroid function of the ovaries in 29 patients with a concentration of $>14 \mathrm{IU} / \mathrm{ml} \mathrm{FSH}$, the risk of early reduction of the ovarian reserve in the case of $100 \%$ is established. Analyzing the coefficients of the discriminant function, EDSS score and total FAS contribute more to discrimination. The developed prognostic model allows to assess the risk of early reduction of ovarian function in women with MS and may be introduced by clinical practice to improve the awareness of doctors and patients about family planning issues.

\section{(c) (i) This work is licensed under Creative}

Submission Link: https://biomedres.us/submit-manuscript.php

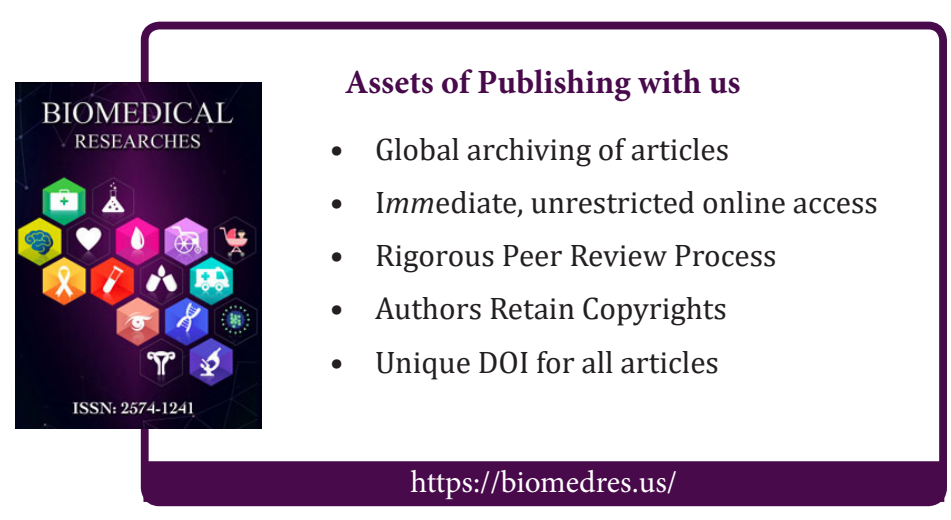

